International Journal of Current Advanced Research

ISSN: O: 2319-6475, ISSN: P: 2319 - 6505, Impact Factor: SJIF: 5.995

Available Online at www.journalijcar.org

Volume 6; Issue 5; May 2017; Page No. 3628-3634

DOI: http://dx.doi.org/10.24327/ijcar.2017.3634.0334

Research Article

\title{
A NOVEL APPROACH FOR IDENTIFYNG DIFFERENT SHAPES BASED ON EDGES USING CLASSIFICATION ALGORITHMS IN IMAGE PROCESSING
}

\author{
Ponnaganti Kalyani and M.Varalakshmi
}

Department of Computer Science \& Engineering, Lingayas

Institute of Management and Technology, A.P., India

\begin{tabular}{l}
\hline A R T I C L E I N F O \\
\hline Article History: \\
Received $19^{\text {th }}$ February, 2017 \\
Received in revised form $12^{\text {th }}$ March, 2017 \\
Accepted $5^{\text {th }}$ April, 2017 \\
Published online $28^{\text {th }}$ May, 2017
\end{tabular}

Key words:

Image Acquisition, Shape Preprocessing, Edge

Detection, Feature Extraction, Image

Thresholding, Shape Identification and Shape

Classification.

\begin{abstract}
A B S T R A C T
A Shape Recognition is one of the basic shape analysis and an important task in the area of computer Vision. Shape recognition has main role in supporting many industrial needs, image processing tasks, classification, etc. The work in this project concentrates on shape recognition which specifically starts from image acquisition, edge detection and classification in image processing. The main emphasis is given for basic shape recognition and classification with the help of the image processing and classification techniques.
\end{abstract}

Copyright $\mathrm{C} 2017$ Ponnaganti Kalyani and M.Varalakshmi. This is an open access article distributed under the Creative Commons Attribution License, which permits unrestricted use, distribution, and reproduction in any medium, provided the original work is properly cited.

\section{INTRODUCTION}

The acquisition and analysis of the visual information produced by the interaction between light and the world objects have represented powerful means through which humans and animals can quickly and efficiently learn about their surrounding environments. In this context, [1] there are several objects, such as characters, which are defined in terms of 2D shapes and should therefore be represented, characterized and processed.

\section{Applications Document Analysis}

WWW, OCR (optical character recognition), multimedia databases, historical documents.

VisualArts: Video restoration, special effects, video tracking.

Internet: watermarking, graphic design and usability.

Neuroscience: Morphological taxonomy of neural cells, investigations, modeling of biologically realistic cells, simulation of neural structures.

Medicine: Tumor recognition, quantification of change and/or deformation of anatomical structures, morphometric analysis for diagnosis

\section{*Corresponding author: Ponnaganti Kalyani}

Department of Computer Science \& Engineering, Lingayas Institute of Management and Technology, A.P., India

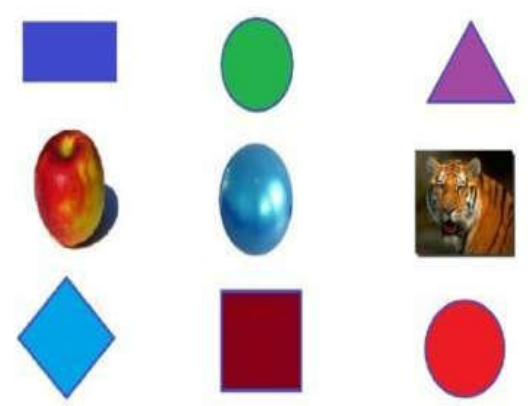

Figure 1 Some typical and easily recognizable 2D shapes

Biology: Morphometric-based evolution comparison, taxonomy, comparative anatomy, cytology, identification and counting of cells (e.g., white blood cells), microscopy.

Physics: Analysis of particle trajectories, crystal growth, polymers.

Engineering: Semiconductors, quality control, danger detection, machine interpretation of line drawings, computer aided design of mechanical parts and buildings, automation, robotics, remote sensing.

Security: Fingerprint/face/iris detection, biometrics, human gait, signature verification.

Agriculture: Harvest control, seed counting and quality control, species identification, fruit maturation analysis. 


\section{Preliminary Concepts}

\section{Computational shape analysis}

Computational shape analysis involves several important tasks, from image acquisition to shape classification.[1]

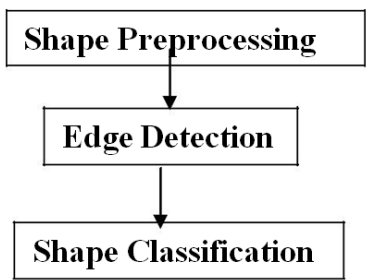

Figure 2 Shape analysis tasks and their organization into three main classes.

Shape acquisition involves acquiring an image (e.g., photograph) and digitizing it, so that it can be properly processed by a computer (Figure 1.3).[4] The type of image acquisition framework and devices to be used depends heavily on the application, e.g., a camera attached to

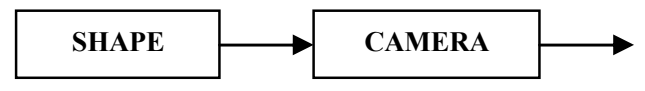

Figure 3 shape acquisition to be processed by aComputer

\section{Shape Acquisition}

Figure 1.6 shows an image that is half black and half white (it could be, for instance, a zoomed detail of an edge region of the binary objects in Figure 1.5(b)). The matrix representation of this digital image is also shown in Figure 1.6, where the black region corresponds to the gray-level 0 and the white region to the gray-level 255 .
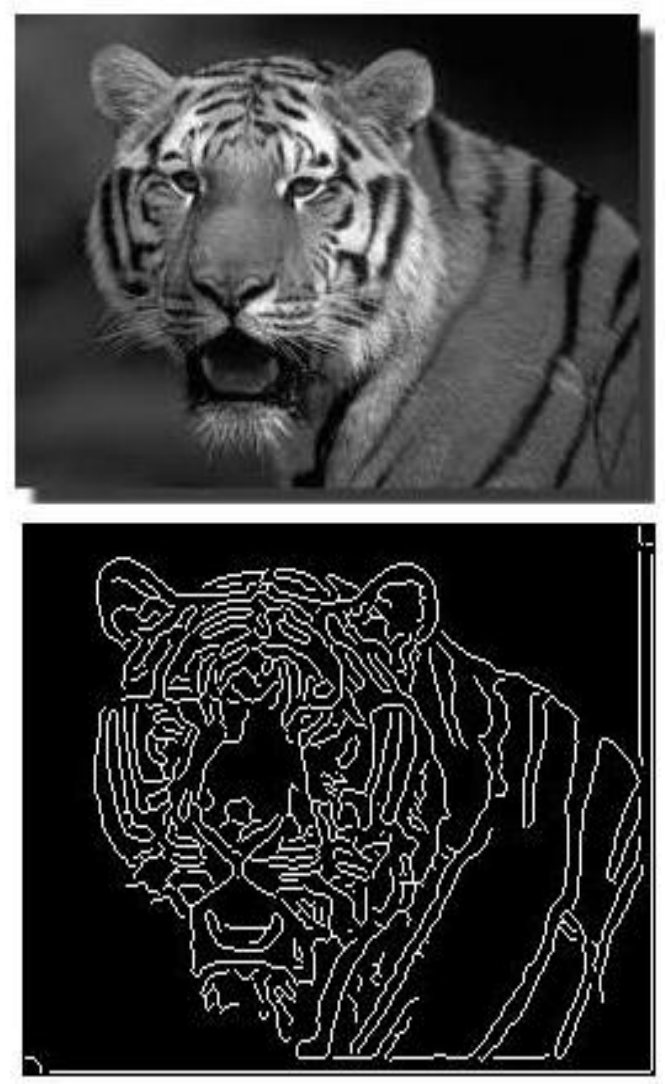

Figure 5 Digital image (a) and respective edge detection (b).

\section{Shape Pre-Processing}

The first step toward the computational morphological analysis of a given object.

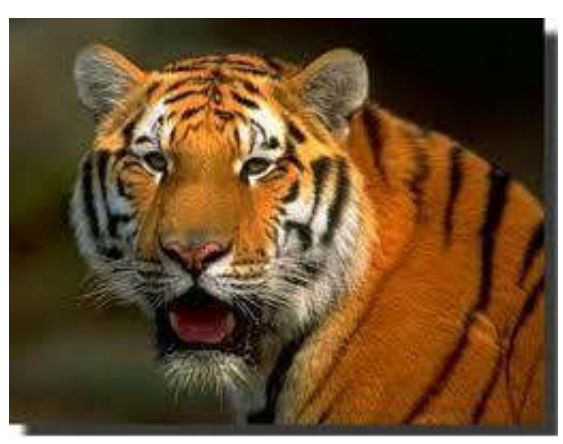

a

\section{Edge Detection}

A subsequent important step in most image analysis approaches is the partitioning of the image in terms of its components, or patches that define the different foreground objects and the background of a scene. In order to perform shape analysis over the image objects (i.e., the leaves), it is necessary

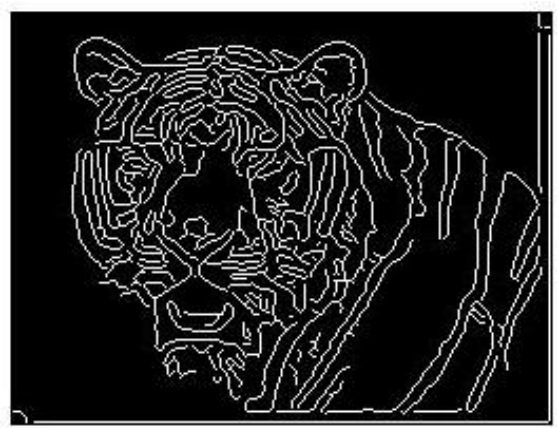

Gray-Level Edge Detection

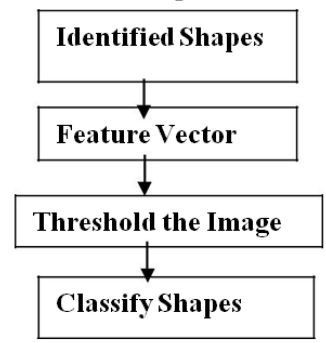

Figure 6 The concept of edge detection through image differentiation.

\section{Classification}

Classification is done particularly in the case of shape analysis as it is important to carefully visualize and inspect the shapes to be classified.

\section{Shape Classification}

Finally, after performing shape preprocessing, and Edge detection classification algorithms are usually applied in order to assign a class to each considered shape. The important aspects related to shape classification is Feature Extraction and Image Thresholding. [9]

\section{Steps involved in classification}

The stages in shape classification are explained in the following steps. 


\section{Feature Extraction}

If the features extracted are carefully chosen it is expected that the features set will extract the relevant information from the input data in order to perform the desired task using this reduced representation instead of the full size input.

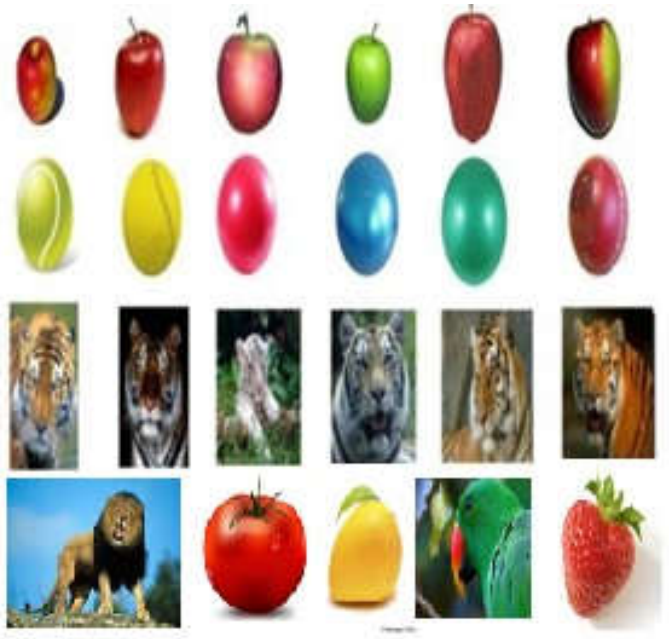

Figure 8 Examples of each of the six classes.

\section{Image Thresholding}

[1]. For instance, in the image, the pixels of the background range from 250 to 255 , while those belonging to the objects range from 0 to 240 .

This is summarized by the following algorithm:

\section{Algorithm: Thresholding}

For each pixel in the image do If (pixel value $<$ threshold) Then Label the pixel as an object pixel; Else

Label the pixel as a background pixel;

\section{Problem Statement and Requirements}

\section{Specification}

\section{Problem Statement}

Existing System: The main features involved are shape preprocessing, edge detection and classification.

There are many systems which are developed using the combination of image preprocessing and edge detection.

There are many systems which are developed using the combination of image preprocessing and edge detection. In this existing system it is identified all types of regular shapes like rectangle, circle, diamond, square, eclipse, etc. and some irreregular shapes like tigers, balls ,apple and combination and classification of these.

Proposed System: In this proposed system, project consists of 3 phases. The $1^{\text {st }}$ phase is a pre process an image. The $2^{\text {nd }}$ phase is a edge detection on basic shapes of an input image using $2 \mathrm{D}$ transformation techniques. The $3^{\text {rd }}$ phase is the extracting the features of the images into its classes.

\section{Requirements Specification Purpose of the system:}

In this proposed system, project consists of 3 phases. The $1^{\text {st }}$ phase is a preprocess an image. The $2^{\text {nd }}$ phase is a edge detection on basic shapes of an input image using 2D transformation techniques. The $3^{\text {rd }}$ phase is the extracting the features of the images into its classes.

\section{Functional Requirements}

1. The Application should work with classes of images of a Database

2. The image should be homogenous and directed along any orientation

3. The images considered are apples, tigers, balls and all regular shapes therefore a Database of apples, tigers, balls and all regular shapes are required

\section{System Environment}

Minimal required Hardware Configuration:

$\begin{array}{ccc}\text { Processor: } & & \text { Pentium IV } \\ \text { Clock Speed } & : & 2.4 \mathrm{GHz} \\ \text { Ram } & : & 512 \mathrm{MB}\end{array}$

Minimal required Software Configuration:

Operating System: Windows XP

Programming Tool: MATLAB R11a

\section{Analysis and Design}

Analysis

The various stages in the Application development are:

- Image Processing and Database Features Generation

- Loading Input Image and Image Conversion

- Identifying the Edges of Objects in Input Image

- Recognizing the Shapes of an Image

- Classification and Result Generation

\section{UML Diagrams}

\section{Use Case Diagram}

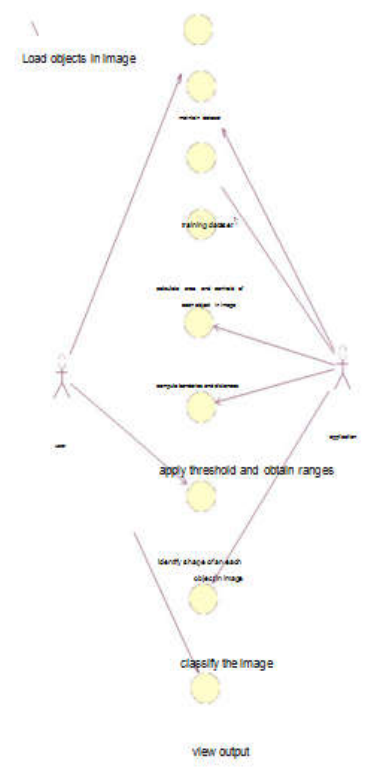

Design

The 3 phases will be carried out as follows: Phase1 Image preprocessing

The query image is converted from RGB to Gray-Scale image by using the MATLAB command rgb2gray. Phase2 Edge detection: 
One of the first steps generally required in shape analysis is to detect the shape. In order to perform shape analysis over the image objects, it is necessary to partition image objects and the background of a scene.

\section{Phase3 Shape identification and classification}

After performing edge detection on objects in image, calculate the centroid and area of each object in image by using the functions $\mathrm{s}=$ regionprops $(\mathrm{L}$, 'centroid') and $\mathrm{DT}=$ regionprops (L, 'area'); in MATLAB.The boundaries of an objects in image is found by using the function boundaries = bwboundaries (BW); Now, we find the distances between the boundaries and centroids by applying the function distance $\{\mathrm{k}\}(1, \mathrm{i})=\operatorname{sqrt}\left((\mathrm{b}(\mathrm{i}, 2)-\mathrm{s}(\mathrm{k}) \cdot \text { Centroid(1) })^{\wedge} 2+\right.$ $\left.(b(i, 1)-s(k) \text {.Centroid(2) })^{\wedge} 2\right)$; then find the maximum and minimum distances by using the functions $\mathrm{a}=\max (\operatorname{distance}\{\mathrm{k}\})$; and $\mathrm{b}=\min ($ distance $\{\mathrm{k}\})$;

After finding the minimum and maximum distances, compute the difference of an each object in image by using the function difference $=a-b$;

Edge detection, 'feature extraxtion', intensity conversions based upon the input, say rgb2gray or mat2gray etc depending upon the file format; GIF or JPEG or PNG etc.If threshold of any recognition image matches with the training work space, recognition as that of that Training Image recognized.

\section{Implementation}

This project is implemented in three phases: Phase 1: Image Preprocessing Phase 2: Edge Detection

Phase 3: Shape Identification and Classification

\section{Phase1: Shape Preprocessing}

This process involves acquiring and storing an objects in image. Shape detection is performed on image analysis.

1. Here eliminate the noise occurred of an objects in image by using the shape operations.

2. Load the images in the training data set.

3. The objects in the image are retrieved will be given as training images for phase 2 , edge detection and phase 3 shape identification and classification.

\section{Phase2: Edge Detection}

1. Identify the sets of points (or curves or contours) corresponding to the more pronounced gray-level variations.

2. Calculate the difference between their respective gray-levels.

3. Apply the canny edge detection technique to detect the edges of an each object in image.

4. The generating result will be given for phase 3, shape identification and classification.

\section{Phase3: Shape Identification and Classification}

Calculate the area and boundaries of the shapes in the image based on the edges obtained after performing phase 2 .

- Compute the distance between the images in the training dataset and the input image by using Euclidean distance.

- Obtain the ranges based on area and boundaries of each image.
- The shapes are compared, and values obtained in step 2 should satisfy the threshold value.

- The shapes in the image which satisfy the above conditions are identified.

- Finally the shapes in the input image can be classified by extracting the features and satisfying the above conditions.

\section{RESULTS}

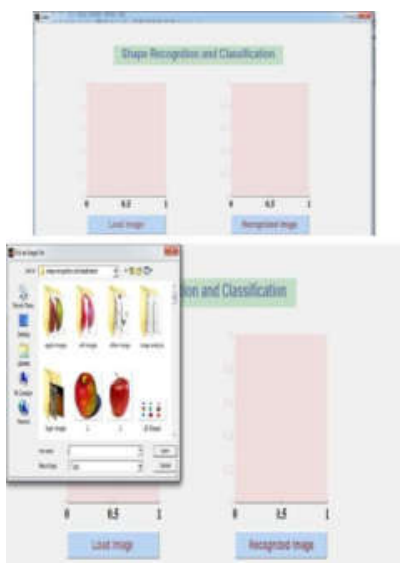

Query Image

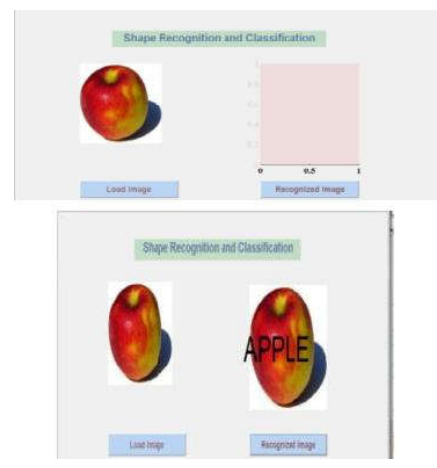

Output Image

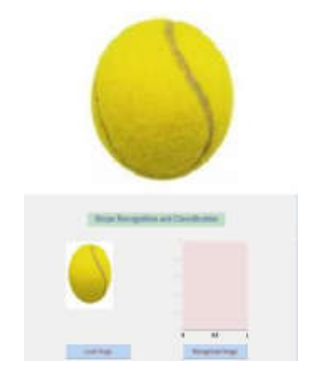

Output

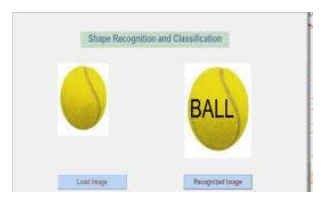

Query Image

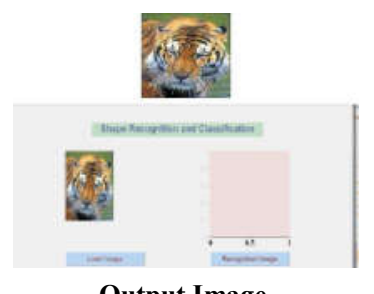

Output Image 


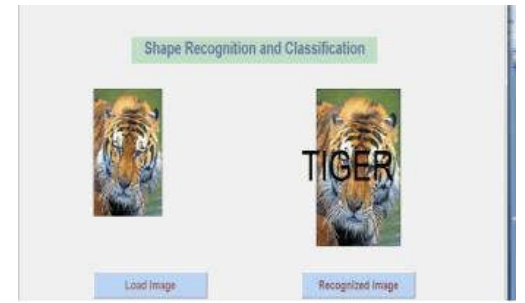

Query Image

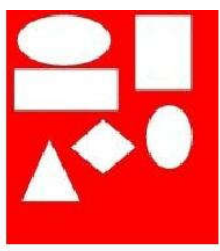

Shape Recagenition and Clesstication

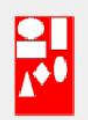

10

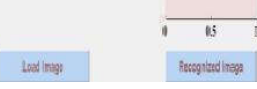

Output Image
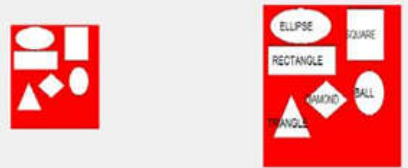

Load inage

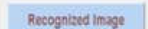

Results of Combination of all Shapes

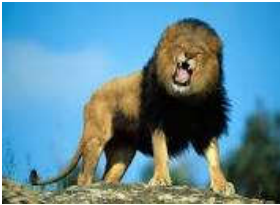

Query Image

Shape Rocognition and Classification
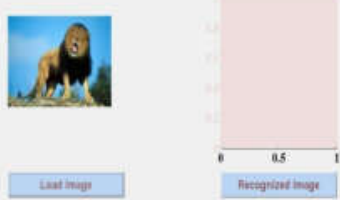

Output Image

Shape Recognition and Classification
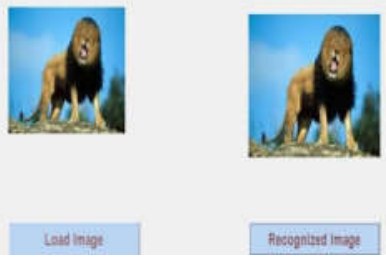

Query Image

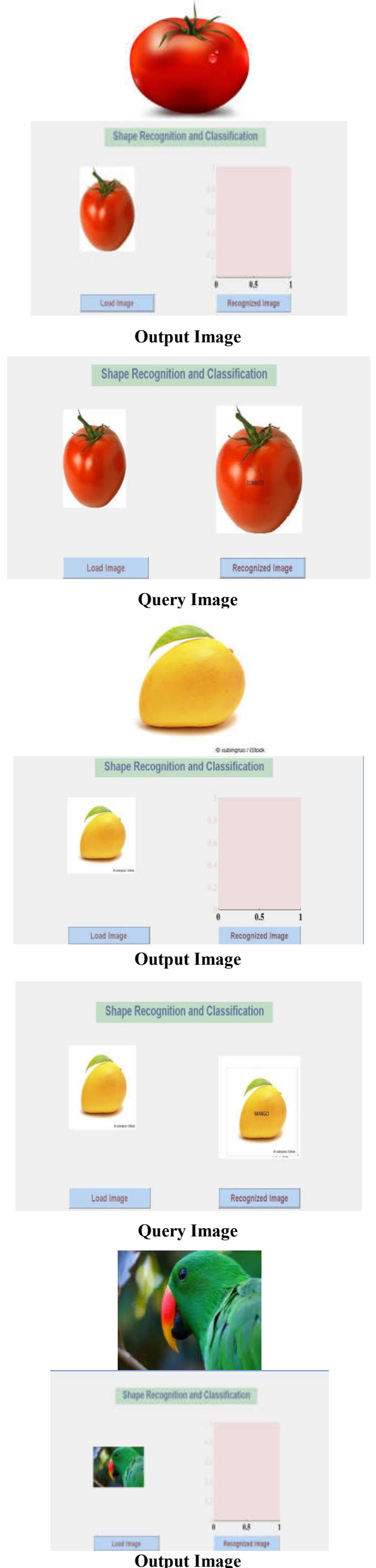

Output Image 


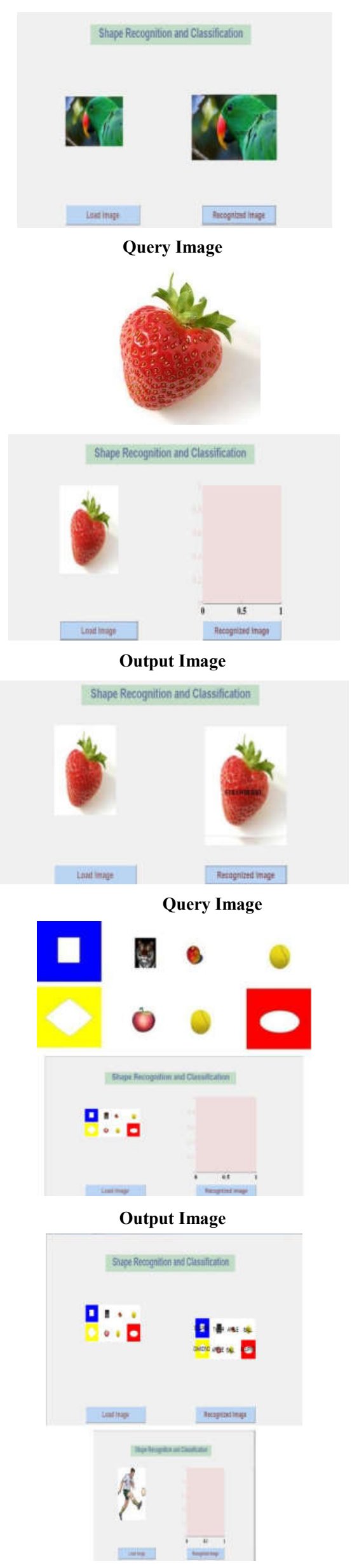

\section{Testing}

Testing Strategy

\section{Unit Testing}

Unit testing refers to tests that verify the functionality of a specific section of code, usually at the function level.

\section{System Testing}

System testing of software or hardware is testing conducted on a complete, integrated system to evaluate the system's compliance with its specified requirements.

\section{Test results: Query Image}

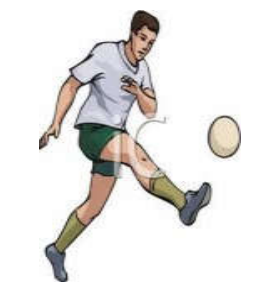

Output Image

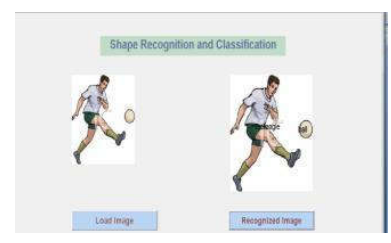

\section{CONCLUSION AND FUTURE WORK}

In this work, we identified all the basic shapes and it has been extended to tiger, parrot, lion, mango, strawberry, and tomato images. Even though the project works well for shapes, it has to be extended for irregular shapes and for other domains also .Even though the methodology used works well for only certain shapes like tiger, parrot, lion, mango, strawberry and tomato, it has to be extended to all other irregular shapes by using enhancing the methodology by using WAVELETS, PCA and other techniques for further improvement and classification.

\section{Bibliography}

1. Luciano Da Fontoura Costa, Roberto Marcondes Cesar Jr., Shape Analysis and Classification: Theory and Practice (Image Processing Series) [Hardcover]

2. http://www.creatis.insa-lyon.fr/ bernard/files/ courses/ DIP-EN_ImageAnalysis_part3.pdf

3. Paul L. Rosin, Further five-point fit ellipse fitting, Graphical Models and Image Processing, v.61 n.5, p.245-259, Sept. 1999 [doi>10.1006/gmip. 1999. 0500]

4. Donnelly N, Found A, Müller HJ (2000) Are shape differences detected in early vision? Visual Cogn 7(6):719-741.

5. Andrew Fitzgibbon, Maurizio Pilu, Robert B. Fisher, Direct Least Square Fitting of Ellipses, IEEE Transactions on Pattern Analysis and Machine Intelligence, v.21 n.5, p.476-480, May 1999 [doi $>10.1109 / 34.765658$ ]

6. Zanker JM, Quenzer T (1999) How to tell circles from ellipses: perceiving the regularity of simple shapes. Naturwissenschaften 86:492-495. 
7. Paul L. Rosin, Measuring rectangularity, Machine Vision and Applications, v.11 n.4, p.191-196, Dec. 1999 [doi>10.1007/s001380050101]

8. O. M. Bruno, R.M. Cesar Jr., L.A. Consularo and L. da F. Costa, Automatic Feature Selection for Biological Shape Classification in SYNERGOS,
9. Proc. Brazilian Conference on Computer Graphics, Image Processing and Vision, (SIBGRAPI-98, Rio de Janeiro - RJ, Out 1998), IEEE Computer Society Press, 363-370, 1998.

10. J. Canny, A Computational Approach to Edge Detection, IEEE Transactions on Pattern Analysis and Machine Intelligence, 8(6):679- 698, 1986. R.M. Cesar Jr. and L. da F. Costa, Shape Characterization by using the Gabor Transform, Proc. 7th IEEE Digital Signal Processing Workshop (Loen, Norway, September 1-4, 1996), 215-218, 1996a.

\section{How to cite this article:}

Ponnaganti Kalyani and M.Varalakshmi (2017) ' A Novel Approach For Identifyng Different Shapes Based On Edges Using Classification Algorithms In Image Processing', International Journal of Current Advanced Research, 06(05), pp. 3628-3634. DOI: http://dx.doi.org/10.24327/ijcar.2017.3634.0334 